\title{
The Use of Artificial Intelligence-Based Optical Remote Sensing and Positioning Technology in Microelectronic Processing Technology
}

\author{
Chenqi Yan $\mathbb{D}$ and Mengchao Tan \\ Tangshan Normal University, Tangshan, Hebei, China \\ Correspondence should be addressed to Chenqi Yan; 2002020320@st.btbu.edu.cn
}

Received 19 August 2021; Accepted 20 October 2021; Published 21 December 2021

Academic Editor: Narasimhan Venkateswaran

Copyright ( 2021 Chenqi Yan and Mengchao Tan. This is an open access article distributed under the Creative Commons Attribution License, which permits unrestricted use, distribution, and reproduction in any medium, provided the original work is properly cited.

\begin{abstract}
The purpose is to make defect detection in microelectronic processing technology fast, accurate, reliable, and efficient. A new optical remote sensing-optical beam induced resistance change (ORS-OBIRCH) target recognition and location defect detection method is proposed based on an artificial intelligence algorithm, optical remote sensing (ORS), and optical beam induced resistance change (OBIRCH) location technology using deep convolutional neural network. This method integrates the characteristics of high resolution and rich details of the image obtained by ORS technology and combines the advantages of photosensitive temperature characteristics in OBIRCH positioning technology. It can be adopted to identify, capture, and locate the defects of microdevices in the process of microelectronic processing. Simulation results show that this method can quickly reduce the detection range and locate defects accurately and efficiently. The experimental results reveal that the ORSOBIRCH target recognition defect location detection method can complete the dynamic synchronization of the IC detection system and obtain high-quality images by changing the laser beam irradiation cycle. Moreover, it can analyze and process the detection results to quickly, accurately, and efficiently locate the defect location. Unlike the traditional detection methods, the success rate of detection has been greatly improved, which is about $95.8 \%$, an increase of nearly $40 \%$; the detection time has been reduced by more than half, from 5.5 days to 1.9 days, and the improvement rate has reached more than $65 \%$. In a word, this method has good practical application value in the field of microelectronic processing.
\end{abstract}

\section{Introduction}

At present, China's manufacturing industry is at a crucial moment of intelligent transformation. The "made in China 2025 " action program proposed by the state points out that China should take intelligent manufacturing as the core, lead a new industrial revolution, guide the development of Chinese manufacturing to automation and intelligence, and move forward from a large manufacturing country to a powerful manufacturing country. "Made in China 2025" emphasizes the need to rapidly promote the deep integration of China's informatization and industrialization, strive to improve the design level and design tools of microelectronic technology dominated by integrated circuits, strive to break through the core chip technology involved in the field of national network information security, and comprehensively improve the national comprehensive strength [1]. Microelectronic technology is high-tech gradually formed and developed based on the continuous miniaturization of traditional electronic circuit systems [2]. Its essence lies in integrated circuits, and it mainly focuses on the research of microintegrated circuits with semiconductors as carriers. Microelectronic devices have the characteristics of lightness, reliability, high speed, high efficiency, and low consumption. Hence, they are widely used in different industries such as national defense, communication, transportation, education, medical treatment, and electronics, which has greatly changed human life [3, 4]. Meanwhile, the integrated circuit technology guided by microelectronic technology becomes increasingly advanced. The defects also increase due to its 
high integration, high complexity, high intensity, and small devices, leading to the difficulty in defect location. The defect location of microelectronic devices usually requires the joint use of various location technologies. The current defect location technology has strong limitations due to its scope of application, and the corresponding location technology can only be selected according to the nature of the defect [5].

In recent years, more detailed and rich optical remote sensing (ORS) images with high resolution appear with the rapid development of ORS technology, so that relevant information can be obtained quickly, efficiently, and widely [6]. At present, the ORS technology has been widely used for target recognition and detection, not only in military border security monitoring and battlefield hostile target locking but also in civil fields such as urban planning and resource detection $[7,8]$. The application obstacles of artificial intelligence (AI) have been broken through $[9,10]$ with the realization of $5 \mathrm{G}$ network technology, which has brought new opportunities to the defect positioning technology in microelectronic processing technology. It can promote the application of ORS technology and rapid positioning technology based on AI in microelectronic processing.

With the AI algorithm as the starting point, how to realize the defect identification, capture, and positioning during microelectronic processing is discussed based on the efficient, fast, detailed, and rich characteristics of ORS target recognition technology and the fast and accurate positioning of optical beam induced resistance change $(\mathrm{OBIRCH})$, so as to reduce energy consumption. This exploration has a certain reference and significance for the application research of AI in the field of microelectronic processing technology.

\section{Materials and Methods}

\subsection{Conceptual Analysis}

2.1.1. AI. The concept of AI was first proposed by John McCarthy in 1956. It was initially defined as science and engineering for manufacturing intelligent machines. New connotation is given with the rapid development of science and technology in the 21st century. At this stage, it is considered that AI is a new technical science for the research and development of theories, methods, technologies, and application systems used to simulate, extend, and expand human intelligence $[11,12]$. As a branch of computer science, AI has been listed as the world's cutting-edge technology together with energy technology, space technology, genetic engineering, and nanoscience since it is put forward.

AI is based on computer science, trying to crack intelligence in essence, and used to create a new intelligent machine with a similar human intelligent response. The specific research contents involve human-computer interaction, machine learning, language and image recognition, and natural language processing [13]. Especially, in recent years, AI has developed rapidly, its application has been expanded in many fields, and fruitful results have been achieved. Hence, it has gradually developed into an independent branch and formed an independent theoretical and practical system. AI not only is applied in computer science but also involves

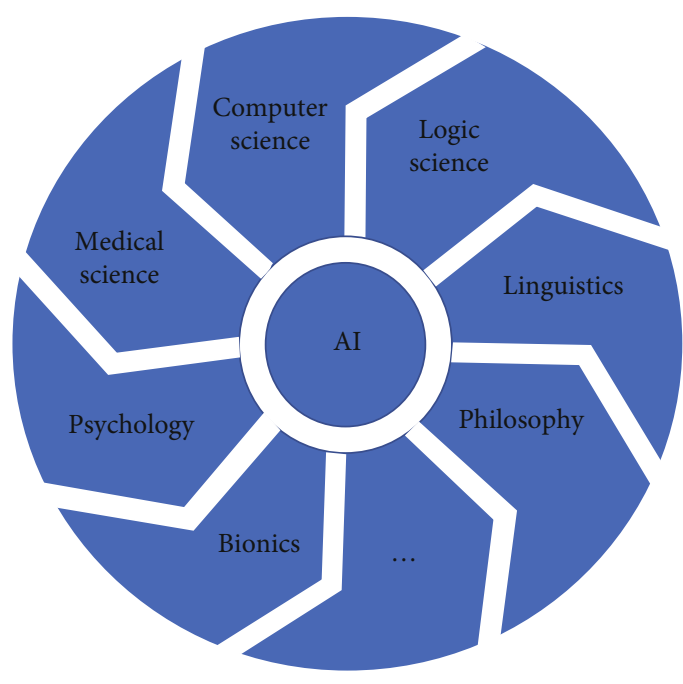

Figure 1: AI science.

many fields such as biology, logic, linguistics, psychology, medicine, and philosophy. It has become a widely crossed front-end science [14], as shown in Figure 1.

2.1.2. ORS. ORS technology is used to identify and judge objects according to their spectral characteristics of light absorption, reflection, and radiation in different bands. Different objects reflect differently in the same band. Even if the objects are the same, they reflect differently in different bands [15]. Various high-performance optical materials appear with the development of science and technology, greatly promoting the progress of ORS technology. The ORS image based on ORS technology can show more details and improve the target accuracy in target detection and recognition because of its high resolution. Besides, it can also promote relevant scientific researchers to observe and study the extraction target more deeply and improve the shortcomings of small coverage and fewer data of traditional observation technology to a certain extent. ORS images have been greatly improved in space, time, and spectral resolution. Since ORS technology has multiple advantages such as high resolution, wide radiation range, and no national boundaries, it has been applied in the fields of people's livelihood such as crop monitoring, disaster assessment, forestry general survey, environmental monitoring, and meteorological forecast. Meanwhile, it is adopted to search, reconnaissance, and track targets in the field of national defense [16-18]. Figure 2 displays its working principle.

2.2. Photoemission Microscope (PEM) Technology. As a new high-resolution microdefect location technology, PEM technology can locate device failure defects quickly and accurately in a wide range, so it is widely used in device failure analysis.

Although the integrated circuit technology has been developed to $14 \mathrm{~nm}$, it is still an effective defect location method. Generally, it is mainly used for defect location in functional failure tests. Its requirement for sample preparation is that it only needs to be opened to expose the wafer surface or the wafer back. Generally, it can globally locate defects in a large range such as a single wafer. Therefore, it 


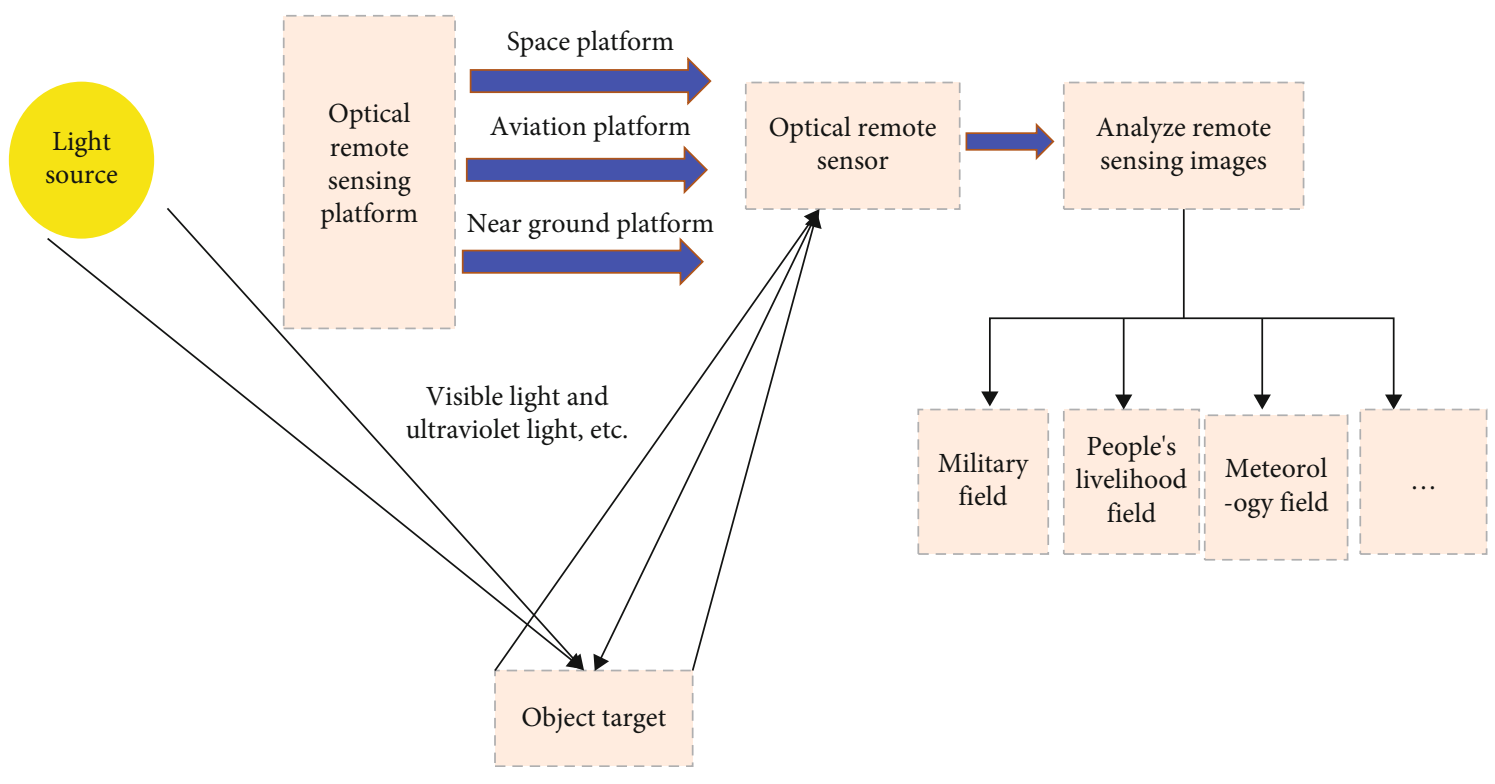

FIGURE 2: Operating principle of ORS.

has the characteristics of nondestructive and global and is an efficient defect location method.

A light emission microscope is a device for detecting the luminescence of semiconductor materials. Figure 3 shows the system composition.

Its working principle is to collect the image of the integrated circuit with the camera and then use the high-sensitivity optical sensor to detect the photons emitted by the integrated circuit when the integrated circuit runs a certain function, so as to form the light emission image. Then, the integrated circuit image and the light emission image are combined into one image, so the specific position of the light-emitting point on the integrated circuit can be known on this final composite image, so as to achieve the purpose of locating defects. The light emission microscope can detect the light-emitting points from the front and back of the integrated circuit.

\subsection{Application of Deep Convolutional Neural Network} (Deep-CNN) in ORS Image Detection and Recognition. ORS target detection and recognition are mainly adopted to solve the problem of "where." This is bound to require the detection and identification algorithm used to eliminate interference and accurately identify. In recent years, the proposal and development of deep learning have set off a frenzy of AI [19]. The traditional machine learning algorithms based on shallow structures are gradually eliminated because of their shortcomings, such as difficult target feature extraction an insufficient resolution and details. Unlike the machine learning of shallow structure, Deep-CNN has stronger advantages in target feature detection and recognition. Its use in the detection and recognition of ORS images has become a new development trend [20]. Deep learning covers a wide range of fields. According to the algorithm, it can be divided into Deep-CNN, deep belief networks (DBN), and deep recurrent neural network (Deep-RNN); according to the dataset, it can be divided into strong supervised learning, weak supervised learning, and semisupervised learning [21]. Figure 4 displays the relationship among deep learning and $\mathrm{AI}$ and machine learning.

Deep-CNN, which is extended based on deep learning, has received extensive attention because of its advantages in ORS image recognition and processing. The commonly used Deep-CNN is extended from the LeNet-5 convolutional neural network [22]. In target detection and recognition in ORS images, the Deep-CNN processing can simplify the algorithm and shorten the time of target feature recognition and extraction. Deep-CNN can automatically recognize target features and has stronger adaptability. Introducing Deep-CNN into ORS technology and applying it to target recognition and detection are a crucial step towards intelligence. At present, target detection and recognition algorithms based on Deep-CNN are roughly divided into two-stage detectors based on Faster Region Convolutional Neural Networks (Faster-RCNN) and single-stage detectors based on You Only Look Once (YOLO) and Single Shot MultiBox Detector (SDD) [23]. Figure 5 shows the process of Deep-CNN target detection and recognition.

The ORS target detection and recognition method based on AI Deep-CNN is introduced into defect detection in microelectronic processing. Microelectronic devices are tiny and dense, so it is difficult to detect and identify their targets. Hence, "dilated convolution" is introduced to ensure the accuracy and recall of target recognition and detection. Dilated convolution has one more parameter of "expansion rate," which is mainly used to define the spacing of convolution kernel values in data processing [24]. It can expand the field of vision; that is, the convolution kernel can be expanded to a certain extent. The equation is as follows:

$$
k^{\prime}=(k-1) * d+1 .
$$




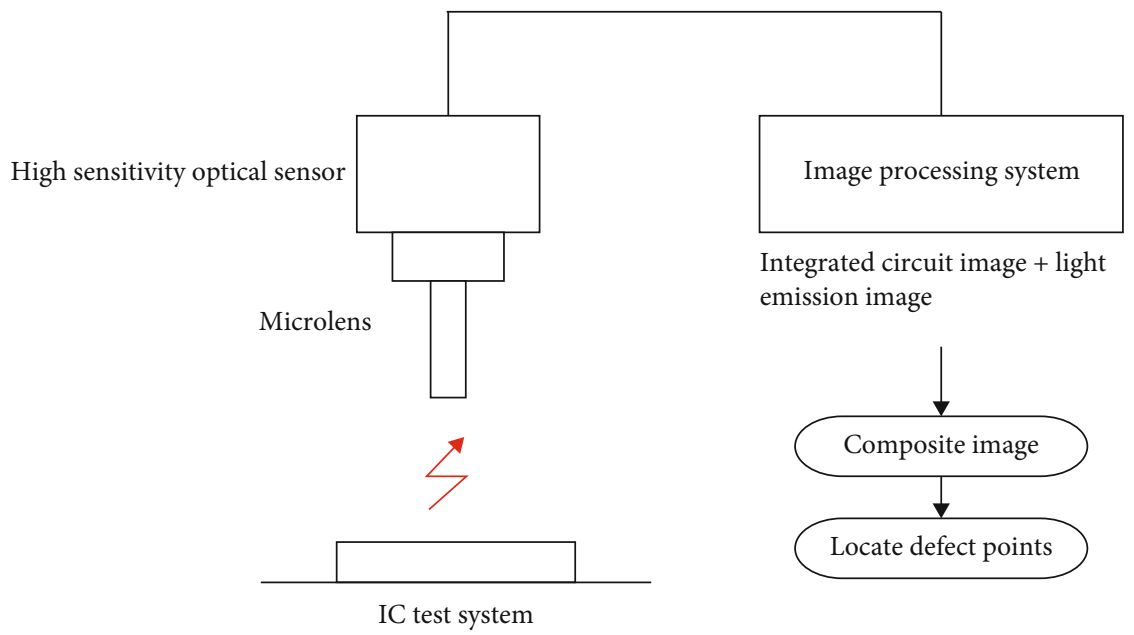

Figure 3: Structure diagram of the light emission microscope system.

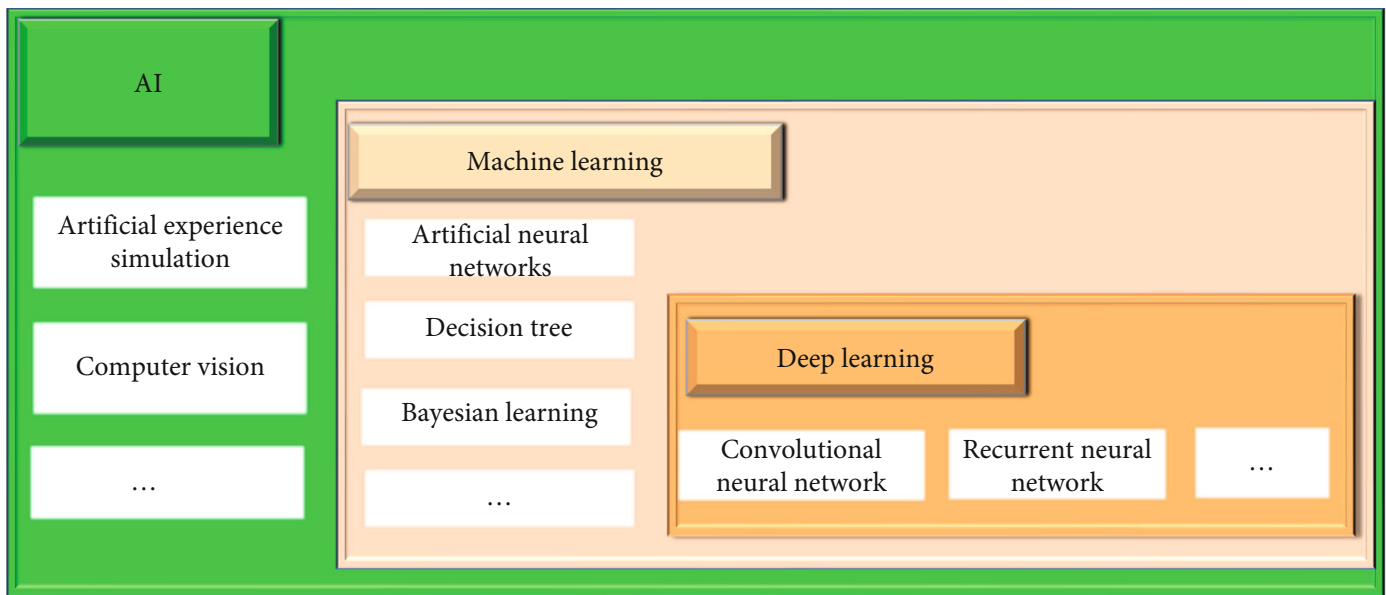

FIgURE 4: Structure relationship of deep learning.

In (1), $k$ is the value of the convolution kernel, $k^{\prime}$ is the value of the processed convolution kernel, and $d$ is the "expansion rate."

In the Region Proposal Network (RPN) stage, the loss function is introduced for training, and the "possible defect point" is set as "normal" and "failure." The judgment is made according to the IoU value between the "possible failure point" collected by the computer and the ideal label. The loss function equation is as follows:

$$
L\left(\left\{p_{i}\right\},\left\{t_{i}\right\}\right)=\frac{1}{N_{\mathrm{cls}}} \sum_{i} L_{\mathrm{cls}}\left(p_{i}, p_{i}^{*}\right)+\lambda \frac{1}{N_{\mathrm{reg}}} \sum_{i} p_{i}^{*} L_{\mathrm{reg}}\left(t_{i}, t_{i}^{*}\right) .
$$

In (2), $\lambda$ is the balance coefficient of multiple tasks, set to 10 , and $p_{i}$ represents the probability of defects at the "possible failure point." $N_{\mathrm{cls}}$ is the size of the training network minbatch, and $N_{\text {reg }}$ is the number of matched anchor frames; $L_{\mathrm{cls}}$ is the classification loss and $L_{\mathrm{reg}}$ is the positioning loss.

$$
L_{\mathrm{cls}}\left(p_{i}, p_{i}^{*}\right)=-\log \left[p_{i}+\left(1-p_{i}^{*}\right)\left(1-p_{i}\right)\right] .
$$

$p_{i}^{*}$ is defined as follows:

$$
p_{i}^{*}=\left\{\begin{array}{l}
1, \\
0 .
\end{array}\right.
$$

In (4), 1 represents that the $i$ th "possible failure point" is defective; 0 means that the $i$ th "possible failure point" is normal.

$t$ is the coordinate regression vector, and the equation is as follows:

$$
\left.\begin{array}{l}
t_{x}=\frac{\left(x-x_{a}\right)}{w_{a}}, t_{y}=\frac{\left(y-y_{a}\right)}{h_{a}}, \\
t_{w}=\log \left(\frac{w}{w_{a}}\right), t_{h}=\log \left(\frac{h}{h_{a}}\right), \\
t_{x}^{*}=\frac{\left(x^{*}-x_{a}\right)}{w_{a}}, t_{y}^{*}=\frac{\left(y^{*}-y_{a}\right)}{h_{a}}, \\
t_{w}^{*}=\log \left(\frac{w^{*}}{w_{a}}\right), t_{h}=\log \left(\frac{h^{*}}{h_{a}}\right) .
\end{array}\right\}
$$




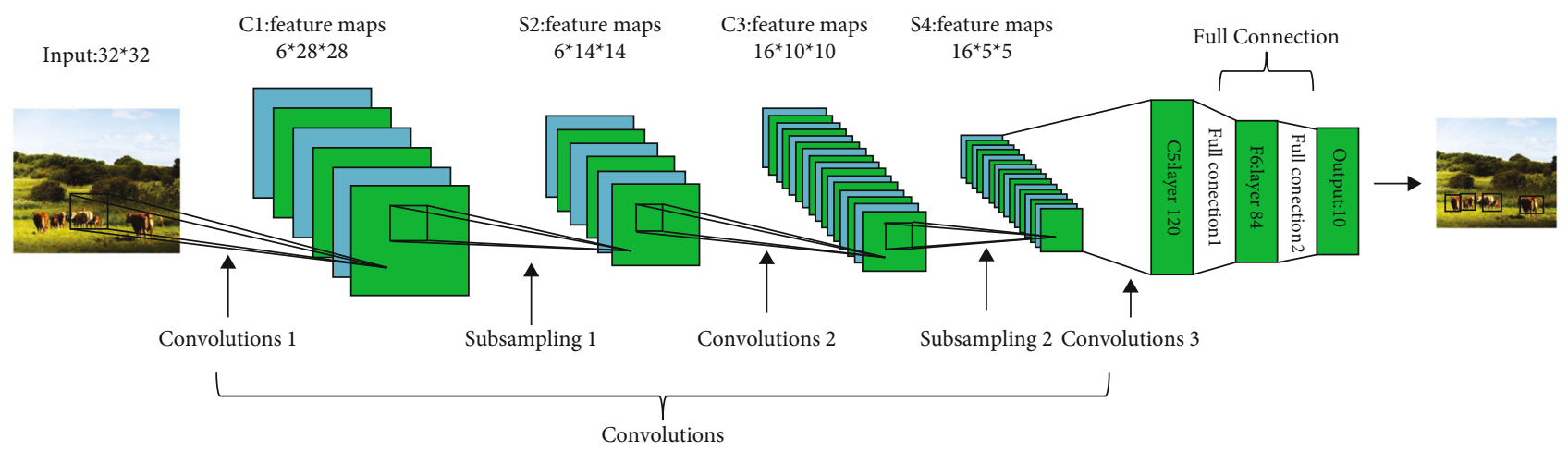

FIgURE 5: Target detection and recognition process of Deep-CNN.

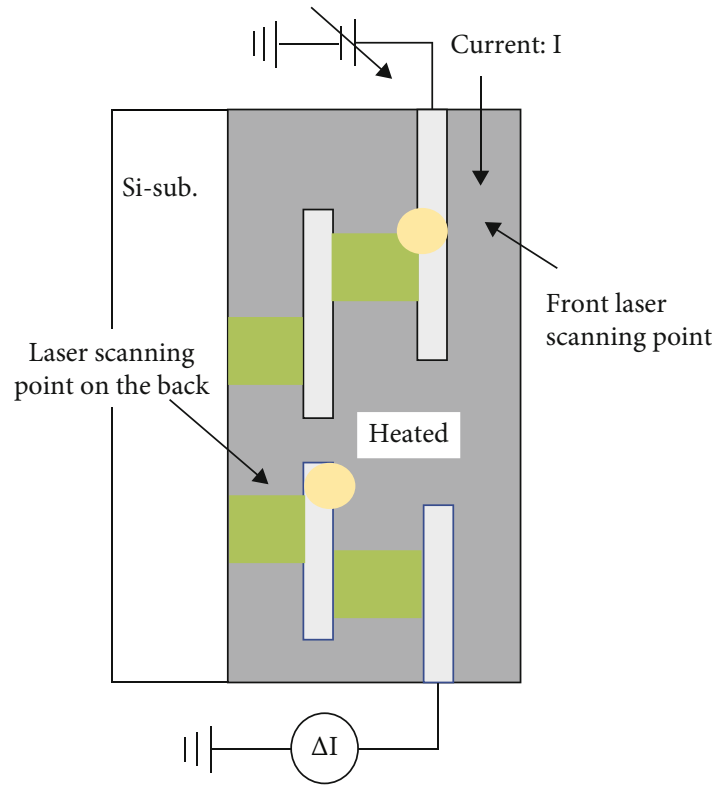

Figure 6: OBIRCH defect location process.

In (5), $w$ and $h$ are the width and height of the prediction frame generated in the RPN stage, respectively; $x$ and $y$ are the central coordinates of the prediction frame generated in the RPN stage; $w_{a}$ and $h_{a}$ are the width and height of "possible failure points" generated in RPN stage; $x_{a}$ and $y_{a}$ are the central coordinates of "possible failure points" generated in RPN stage; $w^{*}$ and $h^{*}$ are the width and height of the ideal coordinate frame; and $x^{*}$ and $y^{*}$ are the center coordinate of the ideal coordinate frame.

At this time, the coordinate regression function is defined as follows:

$L_{\mathrm{reg}}\left(t_{i}, t_{i}^{*}\right)=\sum_{i \in(x, y, w, h)} \operatorname{smooth}_{L 1}\left(t_{i}-t_{i}^{*}\right)= \begin{cases}0.5\left(t_{i}-t_{i}^{*}\right)^{2}, & \left|t_{i}-t_{i}^{*}\right| \leq 1, \\ \left|t_{i}-t_{i}^{*}\right|-0.5, & \text { other. }\end{cases}$

Precision and recall are defined as follows:

$$
\text { Precision }=\frac{\text { Truepositive }}{\text { Truepositive }+ \text { Truenegative }},
$$

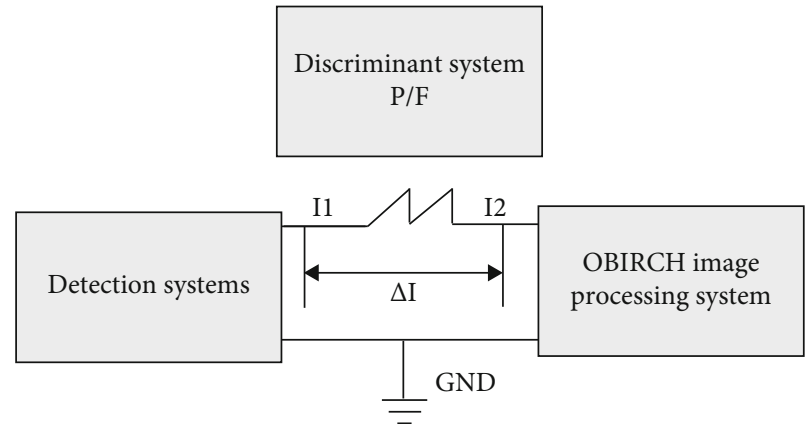

FIgURe 7: Dynamic synchronization diagram of OBIRCH defect location.

$$
\text { Recall }=\frac{\text { Truepositive }}{\text { Truepositive }+ \text { Falsenegative }}
$$

Truepositive is the detected real target, Truenegative is the detected false target, and Falsenegative is the detected false target.

2.4. Analysis of Detecting Integrated Circuit Defects by OBIRCH Technology. OBIRCH positioning technology mainly uses the wavelength of $1.3 \mu \mathrm{m}$ laser beam which heats the integrated circuit, causing the resistance value of a part of the integrated circuit to change under the influence of temperature. The voltage is constant at this time, so the current will change with the change of temperature. The current is detected at this time to locate the defects of the integrated circuit. Semiconductor materials equipped with integrated circuits have similar variation characteristics. Their electrical characteristics are greatly affected by ambient temperature and are more sensitive. Temperature changes can cause changes in the conductivity of semiconductor devices $[25,26]$. This characteristic can be applied to defect location in integrated circuits. Figure 6 displays the defect location process of OBIRCH technology.

The laser beam for positioning access using the OBIRCH method is used to heat the points on the integrated circuit to be measured by point-by-point scanning. The heating time is determined by two factors: the number of lattices in the image of the region and the residence time of the laser beam in the image of the region [27]. Hence, when the OBIRCH positioning system is used, various choices can be made for 
TABLE 1: Experimental platform parameters.

\begin{tabular}{lc}
\hline Device & Parameter \\
\hline Operating system & Linux 16.04 \\
Graphics card & GTX-Tian X \\
CPU & Intel(R) Core (TM) i7-6850K CPU (3.6 GHz) \\
Deep learning framework & Caffe \\
Software & MATLAB2014b/Cudnn5/Opencv3.0 \\
Basic network & VGG16-Net \\
\hline
\end{tabular}

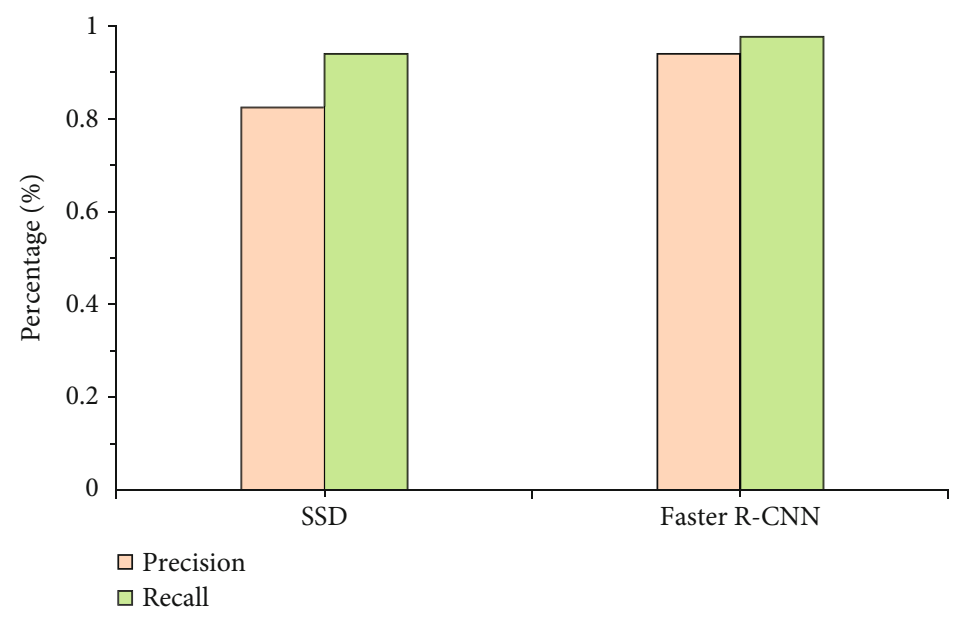

FIgURE 8: Comparison of accuracy and recall of different detection methods.

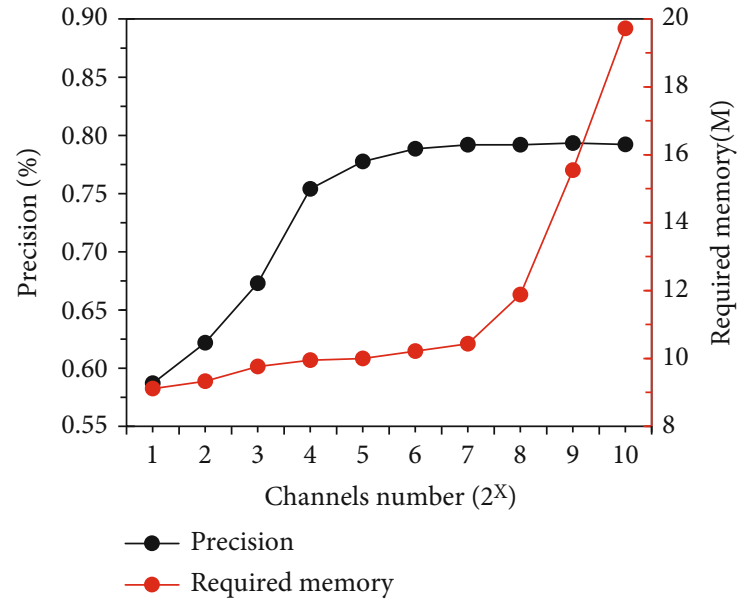

FIGURE 9: Variation curve of accuracy and storage space with the number of convolution kernel channels.

the number of the lattice of the image of the area to be measured and the residence time of the laser beam of the image of the area; besides, the heating time can be flexibly controlled. If the single failure time and result output time during integrated circuit detection are combined as a test cycle, to ensure that the laser can fully heat each point on the image of the detection area and synchronize with the system, it must ensure that the Pixel Dwell Time (PDT) of the laser at each point is not less than twice the test cycle. Only in this

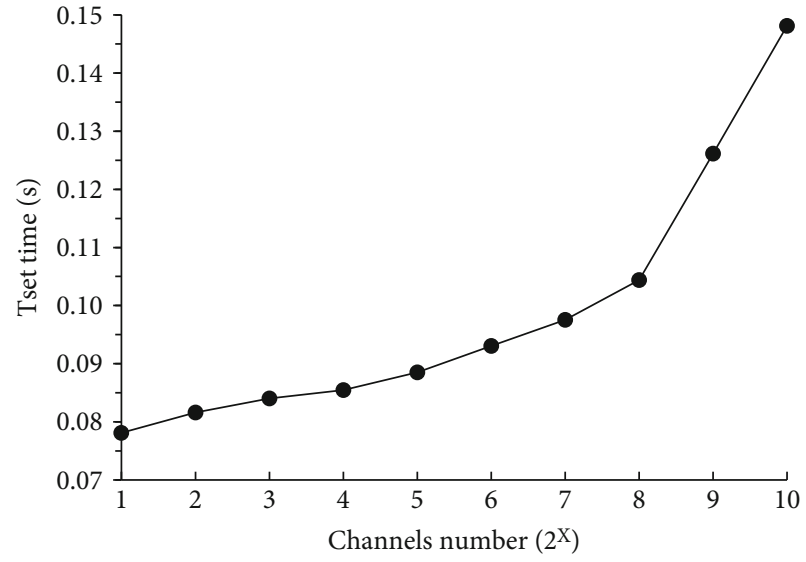

Figure 10: Variation curve of detection time with the number of convolution kernel channels.

way can OBIRCH achieve dynamic synchronization with the integrated circuit test system. Figure 7 is a system diagram.

\section{Experiment and Simulation}

3.1. Construction and Verification of Experimental Platform. Table 1 displays the specific parameters of the ORS platform involved in the platform construction of this experiment.

The performance of the platform should be verified to ensure the accuracy of subsequent experiments, and some ORS chip datasets should be selected for the verification 

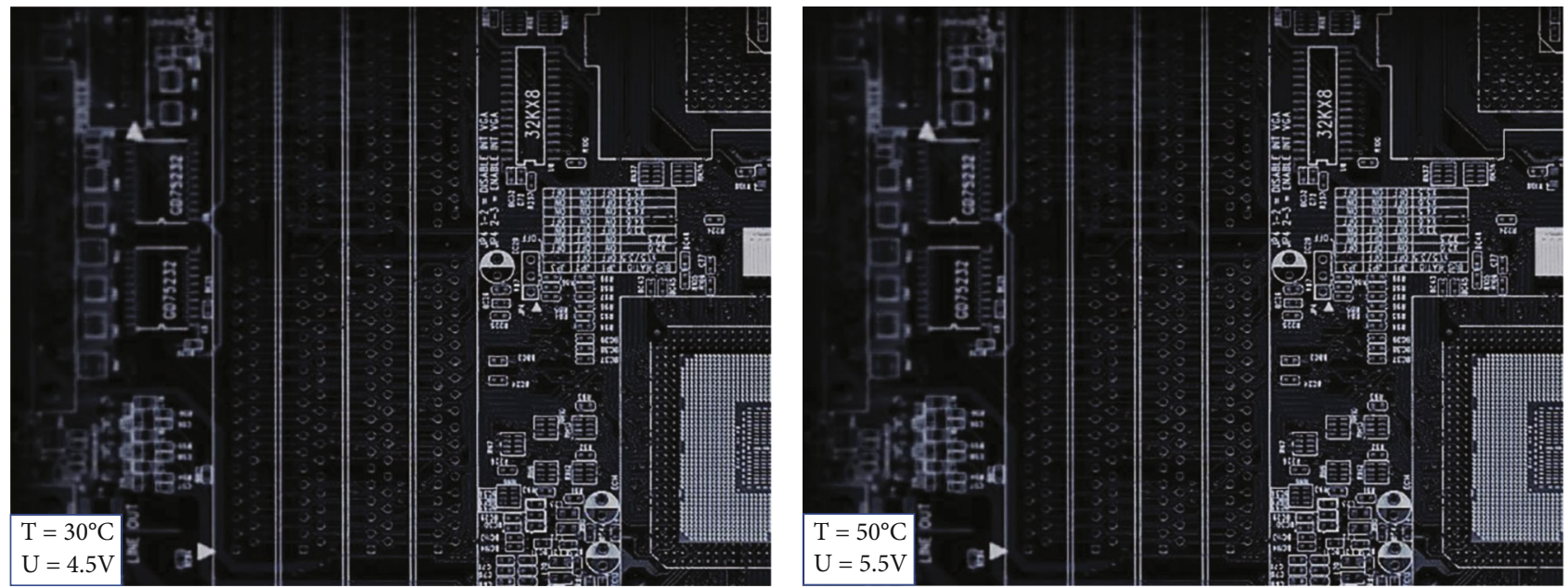

Figure 11: Defect location results of the integrated circuit under PEM detection method.
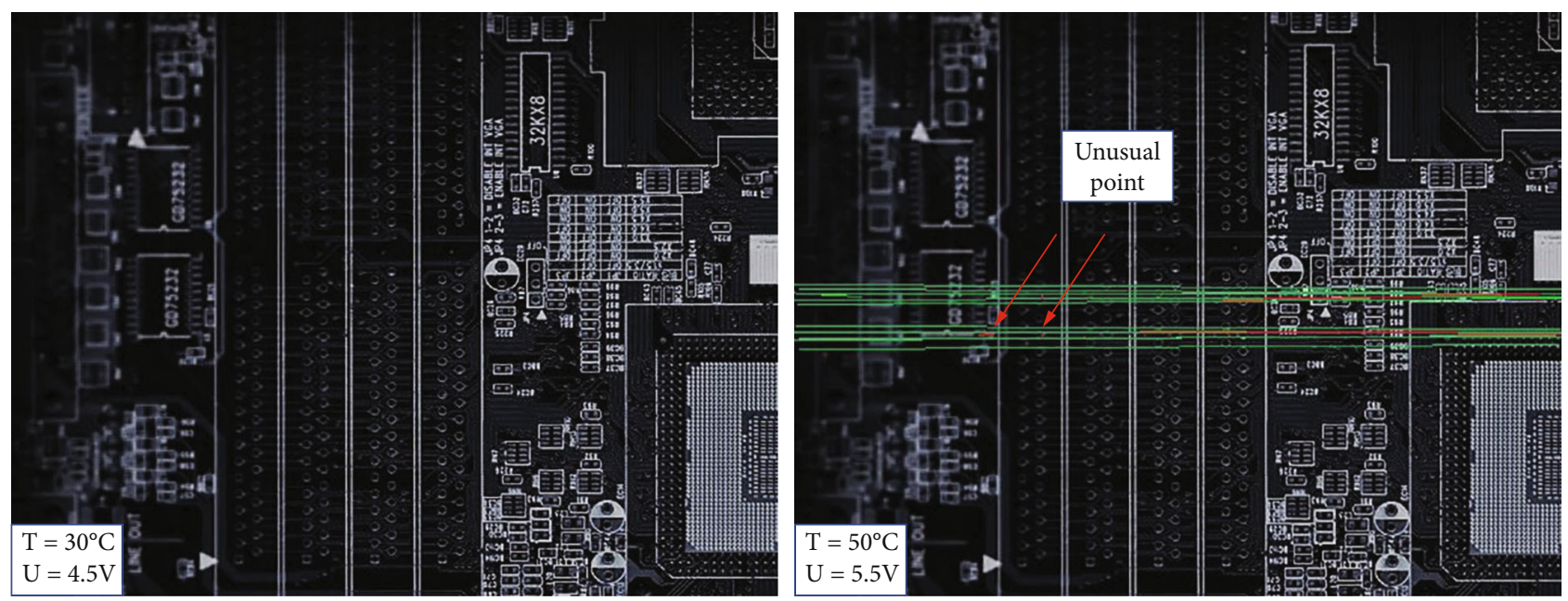

FIGURE 12: Defect location results of the integrated circuit under OBIRCH detection method.

experiment. The dataset has about 300 images and 2000 targets. Faster RCNN is selected for training, with about 80000 iterations. The learning rate and learning momentum of Deep-CNN are set to 0.001 and 0.0005 , respectively. The ORS target detection and recognition method based on AI Deep-CNN is trained and evaluated, and the performance is analyzed by two indexes: precision and recall. Moreover, this method is compared with the traditional SSD target detection model.

\subsection{Experiments of ORS Target Recognition and OBIRCH} Positioning Technology Based on AI Deep-CNN. The microcontroller in microelectronic processing technology is taken as an example to evaluate the effectiveness of ORS target recognition and OBIRCH positioning technology based on Deep-CNN. The failure integrated circuit is a digital integrated circuit based on Complementary Metal Oxide Semiconductor (CMOS) technology, with a size of about $0.7 \mu \mathrm{m}$ and two metal coatings. The detection result is normal when the temperature reaches $30^{\circ} \mathrm{C}$, and the access voltage is between $4 \mathrm{~V}$ and $6 \mathrm{~V}$; the test result shows failure when the temperature exceeds $50^{\circ} \mathrm{C}$, and the access voltage is between $4 \mathrm{~V}$ and $6 \mathrm{~V}$. This failure mode is greatly affected by temperature and access voltage, which is preliminarily speculated to be caused by defects. The above built Deep-CNN ORS target detection and recognition platform is introduced into the experiment and compared with the traditional defect location model of Photoemission Electron Microscopy (PEM).

\section{Results}

4.1. Influence of AI Deep-CNN on ORS Image Detection. The precision and recall of different Deep-CNN target detection and recognition algorithms vary greatly. Figure 8 displays the specific situation.

Figure 8 suggests that in the same dataset, the FasterRCNN algorithm has obvious advantages in accuracy and recall, with accuracy and recall increased by $13.3 \%$ and $3.95 \%$, respectively.

The number of convolution kernel channels exerts different effects on the accuracy, storage space, and detection time of ORS image. Its increase will lead to the change of 


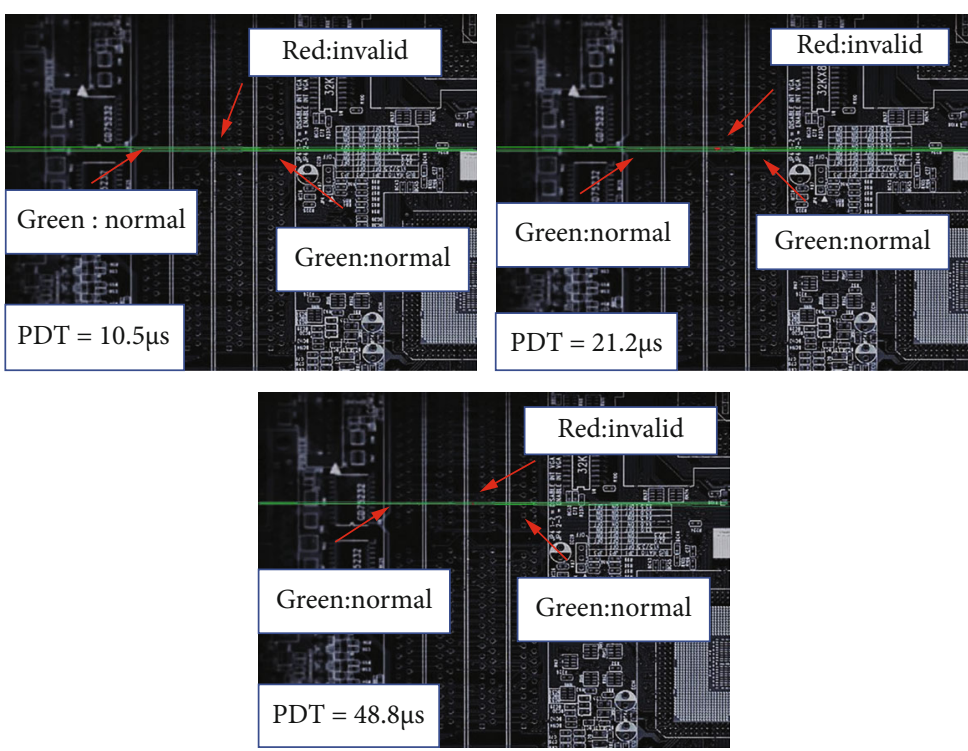

Figure 13: Test results under different test cycles.

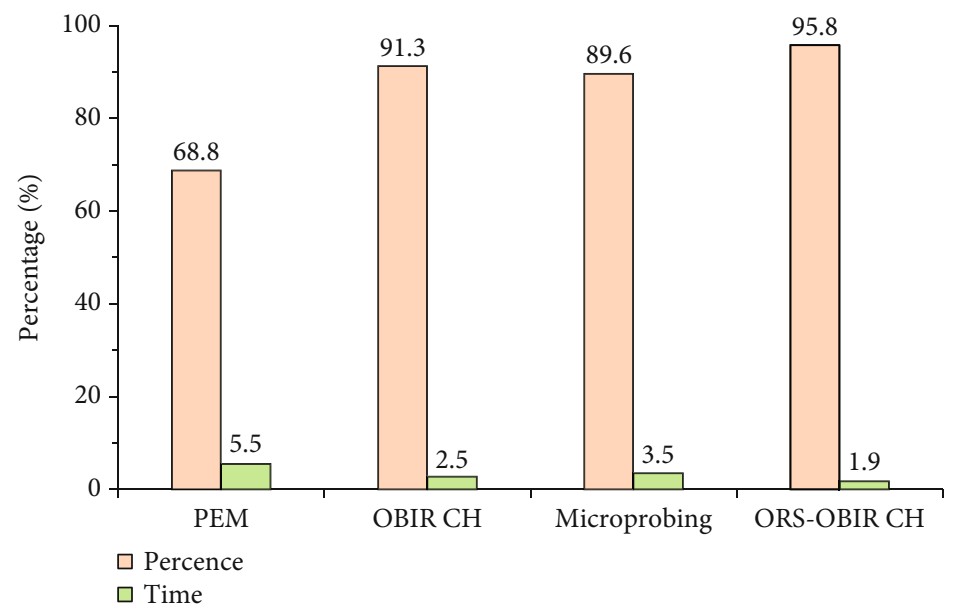

Figure 14: Comparison of different detection methods.

the three. Figure 9 shows the change of ORS image accuracy and required storage space with the number of convolution kernel channels. Figure 10 displays the change of detection time with the number of convolution kernel channels.

Figure 9 displays a positive correlation between the number of channels of the convolution kernel and the accuracy of the ORS image and the required storage space. As the number of channels increases, the image accuracy also increases, but it does not continue to increase when the number of channels is about $2^{6}$; the required storage space continues to increase slightly and increases rapidly when the number of convolution kernel channels is about $2^{7}$. Figure 9 reveals that there is also a positive correlation between the number of channels of the available convolution kernel and the detection time, which quickly increases at $2^{7}$, and the image effect obtained at this time is the best.
4.2. Function Analysis of ORS-OBIRCH Target Recognition and Location Defect Detection Method. Different detection methods have different results caused by laser beam irradiation under different voltage and temperatures, and the performance of failure points is also different. Figure 11 is a detection result obtained using the PEM detection method. Figure 12 is a detection result obtained using the OBIRCH detection method.

Figure 11 suggests that under the traditional PEM detection method, the defects of the integrated circuit cannot be detected under the failure conditions that may be caused by the predetermined voltage and temperature changes; Figure 12 shows that when the OBIRCH detection method is used, the detection effect is poor with a temperature of $30^{\circ} \mathrm{C}$ and an access voltage of $4.5 \mathrm{~V}$; the defect points can be well detected with a temperature of $50^{\circ} \mathrm{C}$ and an access voltage of $4.5 \mathrm{~V}$. 
Different PDTs have different effects on the defect location of integrated circuits. Three different groups of PDT are set up to study its positioning effect. Figure 13 displays the specific results.

Different test cycles are studied under the condition of measured defect location. Figure 13 displays that the positioning point obtained is not very accurate when the laser beam irradiation time is $10.5 \mu \mathrm{s}$, approximately one test cycle; clear positioning points can be obtained when the irradiation time is extended by approximately two cycles to $21.2 \mu$ s, and dynamic synchronization can be achieved at this time; smaller and more accurate positioning points can be obtained when the irradiation time is extended to $48.8 \mu \mathrm{s}$. It suggests that the positioning effect is better with the increase of the heating time of the laser beam to the pixel. Then, different detection methods are compared, as shown in Figure 14.

At present, the success rate and time consumption of several defect detection methods commonly used in the microelectronic integrated circuit industry are compared. Figure 14 shows that the OBIRCH detection method has great advantages in detection success rate. ORS target recognition and OBIRCH positioning technology based on AI Deep-CNN is the most prominent, with a success rate of about $95.8 \%$, which is nearly $40 \%$ higher than the traditional PEM method. Moreover, the detection time is reduced by half, from 5.5 days to 1.9 days, with an improvement rate of more than $65 \%$.

\section{Conclusions}

To sum up, a new ORS-OBIRCH target recognition and location detection method is proposed based on the understanding of the existing AI Deep-CNN, ORS technology, and various integrated circuit defect detection technologies. This method obtains images through ORS technology, which can eliminate interference and obtain images with high resolution and rich details. The verification experiments show that under the same dataset, the Faster-RCNN algorithm has obvious advantages in accuracy and recall, and the accuracy and recall are improved by $13.3 \%$ and $3.95 \%$, respectively; meanwhile, the advantages of photosensitive temperature characteristics in OBIRCH positioning technology are adopted to identify, capture, and locate the defects of microdevices in microelectronic processing. The experimental results show that the ORS-OBIRCH target recognition and location detection method is adopted to detect the defects of integrated circuits in microelectronic processing. The dynamic synchronization of the integrated circuit detection system is completed by changing the laser beam irradiation cycle, high-quality images are obtained, and the detection results of the integrated circuit are processed, which can realize the rapid, accurate, and efficient location of defects. The success rate of detection results has been greatly improved compared with the traditional detection methods, which is about $95.8 \%$, with an increase of nearly $40 \%$; the detection time has been reduced by more than half (from 5.5 days to 1.9 days), and the improvement rate has reached more than $65 \%$. In short, this method can quickly narrow the detection range, locate defects accurately and efficiently, and can be applied in microelectronic processing technology.

\section{Data Availability}

Data are available on request.

\section{Conflicts of Interest}

The authors declare that they have no conflicts of interest.

\section{Acknowledgments}

This study was funded by the Fundamental Research Project of Science and Technology Plan of Tangshan, Study on Planarization Technology of GLSI Multilayer Copper Interconnection, 18130231a, and Doctoral Fund Project of Tangshan Normal University, Study on Planarization Technology of GLSI Multilayer Copper Interconnection, 2018A02.

\section{References}

[1] H. Zhang and J. Han, "Mathematical models for information classification and recognition of multi-target optical remote sensing images," Open Physics, vol. 18, no. 1, pp. 951-960, 2020.

[2] L. F. Chen, "Application analysis of microelectronics technology in smart power consumption," China New Telecommunications, vol. 22, no. 5, p. 97, 2020.

[3] M. Deng, "The specific application of microelectronic control electromechanical equipment in industry," Electronic World, vol. 8, no. 590, pp. 152-153, 2020.

[4] X. Fan, G. Nie, C. Xia, and J. Zhou, "Estimation of pixel-level seismic vulnerability of the building environment based on mid-resolution optical remote sensing images," International Journal of Applied Earth Observation and Geoinformation, vol. 101, no. 3, article 102339, 2021.

[5] Y. Z. Fei, "Research on integrated circuit application and failure analysis method," Microprocessors, vol. 1, no. 41, pp. 1921, 2020.

[6] M. J. DeWeert, A. N. Acker, R. Noguchi, D. Yoon, and G. Sawai, "Principles of surface-phase-resolved shearography," Optical Engineering, vol. 58, no. 11, p. 1, 2019.

[7] X. Hou, Q. Gao, R. Wang, and X. Luo, "Satellite-borne optical remote sensing image registration based on point features," Sensors, vol. 21, no. 8, p. 2695, 2021.

[8] A. V. Kavitha, A. Srikrishna, and C. Satyanarayana, "A review on detection of land use and land cover from an optical remote sensing image," IOP Conference Series Materials Science and Engineering, vol. 1074, no. 1, p. 2002, 2021.

[9] Z. C. Zhang, "Analysis of 5G mobile communication network planning in smart cities," Smart City Applications, vol. 4, no. 2, p. 39, 2021.

[10] H. M. Zhu, "On the development trend of financial reporting in the 5G artificial intelligence era," Economic Management Digest, vol. 754, no. 16, pp. 161-162, 2020.

[11] W. Yang, Y. Wang, Y. Wang, C. Ma, and Y. Ma, "Retrospective deformation of the Baige landslide using optical remote sensing images," Landslides, vol. 17, no. 3, pp. 659-668, 2020. 
[12] H. J. Nasiha, P. Shanmugam, and R. Sundaravadivelu, "Estimation of sediment settling velocity in estuarine and coastal waters using optical remote sensing data," Advances in Space Research, vol. 63, no. 11, pp. 3473-3488, 2019.

[13] P. Cecula, "Artificial intelligence: the current state of affairs for AI in pregnancy and labour," Journal of Gynecology Obstetrics and Human Reproduction, vol. 50, no. 7, article 102048, 2021.

[14] O. M. Korobeynikova, D. A. Korobeynikov, L. V. Popova, T. A. Chekrygina, and E. S. Shemet, "Artificial intelligence for digitalization of management accounting of agricultural organizations," IOP Conference Series: Earth and Environmental Science, vol. 699, no. 1, p. 012049, 2021.

[15] L. Li, G. Zhang, Y. Jiang, and X. Shen, “An improved on-orbit relative radiometric calibration method for agile highresolution optical remote-sensing satellites with sensor geometric distortion," IEEE Transactions on Geoscience and Remote Sensing, vol. 1, no. 99, pp. 1-15, 2021.

[16] K. Topouzelis, D. Papageorgiou, G. Suaria, and S. Aliani, "Floating marine litter detection algorithms and techniques using optical remote sensing data: a review," Marine Pollution Bulletin, vol. 1, no. 170, article 112675, 2021.

[17] F. A. Azizan, A. M. Kiloes, I. S. Astuti, and A. Abdul Aziz, "Application of optical remote sensing in rubber plantations: a systematic review," Remote Sensing, vol. 13, no. 3, p. 429, 2021.

[18] H. Letu, K. Yang, T. Y. Nakajima et al., "High-resolution retrieval of cloud microphysical properties and surface solar radiation using Himawari-8/AHI next-generation geostationary satellite," Remote Sensing of Environment, vol. 239, no. 111583, pp. 111583-111516, 2020.

[19] Y. Li, X. Y. Liu, H. Q. Zhang, X. Li, and X. Sun, "Optical remote sensing image retrieval based on convolutional neural networks," Optics and Precision Engineering, vol. 26, no. 1, pp. 200-207, 2018.

[20] Y. Cheng, P. C. Zhou, and J. W. Han, "High-resolution optical remote sensing image target detection based on rotation invariant convolutional neural network," Science Observation, vol. 15, no. 6, pp. 79-80, 2020.

[21] E. Babaeian, S. Paheding, N. Siddique, V. K. Devabhaktuni, and M. Tuller, "Estimation of root zone soil moisture from ground and remotely sensed soil information with multisensor data fusion and automated machine learning," Remote Sensing of Environment, vol. 260, no. 1, article 112434, 2021.

[22] K. Xu and S. Gao, "Comparison and application analysis of deep convolutional neural networks LeNet-5 and ResNet," Electronic Design Engineering, vol. 28, no. 424, 2020.

[23] B. Wang, Z. M. Zhu, and T. B. Tang, "An improved LeNet-5 convolutional neural network algorithm," Popular Science and Technology, vol. 22, no. 254, pp. 6-8, 2020.

[24] T. Liu and X. L. Wang, "Single-stage target detection using convolution kernel pyramid and cavity convolution," Journal of Image and Graphics, vol. 25, no. 1, pp. 102-112, 2020.

[25] M. Tom, R. Prabha, T. Wu, E. Baltsavias, L. Leal-Taixé, and K. Schindler, "Ice Monitoring in Swiss Lakes from Optical Satellites and Webcams using Machine Learning," Remote Sensing, vol. 12, no. 21, p. 3555, 2020.
[26] Y. Gong, C. Q. Huang, and Y. Liu, "Fault location method of integrated circuit multilayer interconnection structure," Chinese Journal of Chinese Electron Microscopy Society, vol. 39, no. 208, pp. 112-119, 2020.

[27] J. Spurbeck, M. K. Jah, D. Kucharski, J. C. S. Bennett, and J. G. Webb, "Near Real Time Satellite Event Detection, Characterization, and Operational Assessment Via the Exploitation of Remote Photoacoustic Signatures," The Journal of the Astronautical Sciences, vol. 68, no. 1, pp. 197-224, 2021. 